\title{
Near-Optimal Truthful Spectrum Auction Mechanisms With Spatial and Temporal Reuse in Wireless Networks
}

\author{
He Huang ${ }^{1} \quad$ Yu-e Sun ${ }^{2 *} \quad$ Xiang-Yang Li ${ }^{3,4} \quad$ Zhili Chen $^{5} \quad$ Wei Yang $^{5} \quad$ Hongli Xu $^{5}$ \\ ${ }^{1}$ School of Computer Science and Technology, Soochow Univ., Suzhou, China \\ ${ }^{2}$ School of Urban Rail Transportation, Soochow Univ., Suzhou, China \\ ${ }^{3}$ Dept. of Computer Science, Illinois Institute of Technology, USA \\ ${ }^{4}$ Tsinghua National Laboratory for Information Science and Technology (TNLIST), Tsinghua Univ., China \\ ${ }^{5}$ Dept. of Computer Science and Technology, Univ. of Science and Technology of China, China \\ \{huangh, sunye12\}@suda.edu.cn, xli@cs.iit.edu, \{zlchen3,qubit,xuhongli\}@ustc.edu.cn
}

\begin{abstract}
In this work, we study spectrum auction problem where each spectrum usage request has spatial, temporal, and spectral features. After receiving bid requests from secondary users, and possibly reserve price from primary users, our goal is to design truthful mechanisms that will either optimize the social efficiency or optimize the revenue of the primary user. As computing an optimal conflict-free spectrum allocation is an NP-hard problem, in this work, we design near optimal spectrum allocation mechanisms separately based on the techniques: derandomized allocation from integer programming formulation, and its linear programming (LP) relaxation. We theoretically prove that 1) our derandomized allocation methods are monotone, thus, implying truthful auction mechanisms; 2) our derandomized allocation methods can achieve a social efficiency or a revenue that is at least $1-\frac{1}{e}$ times of the optimal respectively; Our extensive simulation results corroborate our theoretical analysis.
\end{abstract}

\section{Categories and Subject Descriptors}

C.2.1 [Computer Systems Organization]: Computer Communication Networks; D.2.8 [Management of Computing and Information Systems]: Installation Management—Pricing and resource allocation

\section{Keywords}

Spectrum auction; Truthful; Approximation mechanism; Social efficiency; revenue

\section{INTRODUCTION}

The growing demand for limited spectrum resource poses a great challenge in spectrum allocation and usage. One of the most promising methods is spectrum auction, which provides sufficient incentive for primary user (a.k.a seller) to sublease spectrum to secondary users (a.k.a buyers). The design of spectrum auction mech-

*Yu-e Sun is the corresponding author.

Permission to make digital or hard copies of all or part of this work for personal or classroom use is granted without fee provided that copies are not made or distributed for profit or commercial advantage and that copies bear this notice and the full citation on the first page. Copyrights for components of this work owned by others than ACM must be honored. Abstracting with credit is permitted. To copy otherwise, or republish, to post on servers or to redistribute to lists, requires prior specific permission and/or a fee. Request permissions from permissions@acm.org.

MobiHoc'13, July 29-August 1, 2013, Bangalore, India.

Copyright 2013 ACM 978-1-4503-2193-8/13/07 ...\$15.00. anisms are facing two major challenges. First, spectrum channels can be reused in spatial, temporal, and spectral domain if the buyers are conflict-free with each other, and thus, allocating the requests of buyers in channels optimally is often an NP-hard problem. Second, truthfulness is regarded as one of the most critical properties, however, it's difficult to ensure truthfulness in a spectrum auction mechanism with performance guarantee. Recent years, many mechanisms were proposed to address some of the auction challenges $[1-3,7-11]$. However, to the best of our knowledge, there is no truthful spectrum auction mechanism with performance guarantee, in which spectrums can be reused both in spatial and temporal domains.

Maximization of the social efficiency, i.e. allocating a channel to buyers who value it most, and maximization of the expected revenue, i.e. allocating a channel to buyers who pay it most, both are the natural goals for spectrum auctions. Thus, we design a framework for spectrum auction which can maximize the social efficiency or the expected revenue. Since channels can be reused in both spatial and temporal domain, the problem of allocating requests of buyers in channels optimally to maximize the social efficiency or the expected revenue is NP-hard. To tackle this challenge, we first relax the integer programming formulation of the channel allocation problem into a linear program (LP) problem, which is solvable in polynomial time. A fractional solution for channel allocation can be obtained by solving this LP optimally. Then, we transfor$\mathrm{m}$ this fractional solution into a feasible integer solution of the original channel allocation problem by using a carefully designed randomized rounding procedure that ensures the feasibility of the solution and good approximation to the objective functions. We prove that the expected weight of the feasible integer solution is at least $1-1 / e$ times of the weight of the optimal solution. To complete our allocation mechanism, we also propose a derandomization algorithm to get a feasible solution whose weight is always guaranteed to be at least $1-1 / e$ times of the weight of the optimal solution. Then, we propose a revised derandomization algorithm and prove that this new allocation method does satisfy the bid-monotone property, thus, implying a truthful mechanism. We point out that our allocation mechanisms can either approximate the social efficiency or the expected revenue, but not both simultaneously.

\section{PRELIMINARIES}

Auctions in our model are executed periodically. In each round, the primary user subleases the access right of $m$ channels in the fixed areas during time interval $[0, T]$, and $n$ buyers request the usage of channels in fixed time intervals and geographical location- 
s/areas. Our goal is to allocate these requests of buyers in channels, such that we maximize either the social efficiency or the expected revenue. We consider two models of the requests of buyers. The first one is the Point model, in which each buyer requests the usage of channels in a particular geographical location and during a fixed time interval. The second one is Area model, in which each buyer requests the usage of channels in a particular geographical area and also during a fixed time interval.

We use $\mathcal{S}$ to denote the set of channels, and define each channel $s_{j} \in \mathcal{S}$ as $s_{j}=\left(R_{j}, A_{j}\right)$, where $A_{j}$ is its license area, and $R_{j}$ is the interference radius of a transmission when a user transmits in channel $s_{j}$. Let $\mathcal{B}$ be the set of buyers, in which each buyer $i \in \mathcal{B}$ is assumed to have a request $r_{i}$. Let $\mathcal{R}$ be the set of requests of buyers. Each request $r_{i} \in \mathcal{R}$ is defined as $r_{i}=\left(L_{i}, b_{i}, v_{i}, a_{i}, t_{i}, d_{i}\right)$, where $L_{i}$ is $i$ 's geographical location in Point model or the area where $i$ wants to access the channel in the Area model, $b_{i}$ denotes its bidding price, $v_{i}$ stands for its true valuation, and $a_{i}, d_{i}$, and $t_{i}$ denote the arrival time, deadline, and duration time (or time length), respectively. In this paper, we only consider the case of $d_{i}-a_{i}=t_{i}$, which means that the time request from the buyer is a fixed time interval. We leave the case of $d_{i}-a_{i}>t_{i}$ as the future work.

We say that two requests $r_{i}$ and $r_{k}$ conflict with each other, if they satisfy the following constrains: (1) The distance between $L_{i}$ and $L_{k}$ is smaller than twice of the interference radius in the Point model, or $L_{i} \bigcap L_{k} \neq \emptyset$ in the Area model; and (2) The required time intervals from $r_{i}$ and $r_{k}$ overlap with each other. We denote the conflict relationships among requests by a conflict graph $\mathcal{G}=(\mathcal{V}, \mathcal{E})$, where $\mathcal{V}$ is the set of requests of buyers, and edge $\left(r_{i}, r_{k}\right) \in \mathcal{E}$ if requests $r_{i}$ and $r_{k}$ conflict with each other. Note that, for the same requests $r_{i}$ and $r_{k}$, different interference radii of channels will lead to a different conflict relationship. We use a matrix $Y=\left(y_{i, k, j}\right)_{n \times n \times m}$ to represent the conflict relationships in graph $\mathcal{G}$, in which if requests $r_{i}$ and $r_{k}$ conflict with each other in channel $s_{j}, y_{i, k, j}=1$; otherwise, $y_{i, k, j}=0$. Since the spectrum is a local resource, we also need to define a location matrix $C=\left(c_{i, j}\right)_{n \times m}$ to represent whether $L_{i}$ is in the license regions of channel $s_{j} . c_{i, j}=1$ if $L_{i}$ is in the license regions of channel $c_{j}$; otherwise, $c_{i, j}=0$. Therefore, two requests $r_{i}$ and $r_{k}$ can share channel $s_{j}$ only if $y_{i, k, j}=0$, and $c_{i, j}=1, c_{k, j}=1$.

The objective of our work is to design a mechanism satisfying truthfulness constraint, while maximizing the social efficiency or revenue. In our problem definition, an auction is said to be truthful if revealing true valuation is the dominant strategy for each bidder, regardless of other bidders' bids. An auction mechanism is truthful if its allocation algorithm is monotonic and it always charges critical values from its buyers [5]. The critical value for a buyer is the minimum bid value, with which the buyer will win the auction.

Social Efficiency Maximization: Social efficiency for an auction mechanism is defined as $\max \sum_{r_{i} \in \mathcal{R}} v_{i} x_{i}$, where $x_{i}=1$ if buyer $i$ wins in the auction; otherwise, $x_{i}=0$.

Revenue Maximization: The revenue of an auction is the total payment of buyers. An auction maximizing the revenue for the auctioneer is known as an optimal auction in economic theory [4]. In the optimal auction, Myerson introduces the notion of virtual valuation $\phi_{i}\left(b_{i}\right)$ as

$$
\phi_{i}\left(b_{i}\right)=b_{i}-\frac{1-F_{i}\left(b_{i}\right)}{f_{i}\left(b_{i}\right)}
$$

where $F_{i}\left(b_{i}\right)$ is the probability distribution function of true valuations of buyer $i$, and $f_{i}\left(b_{i}\right)=\frac{d F_{i}\left(b_{i}\right)}{d b_{i}}$. According to the theory of optimal auction [4], maximizing the expected revenue is equivalent to finding the optimal solution of $\max \sum_{r_{i} \in \mathcal{R}} \phi_{i}\left(b_{i}\right) x_{i}$.

\section{A STRATEGYPROOF SPECTRUM AUC- TION FRAMEWORK}

In this section, we propose a general truthful spectrum auction framework with the goal of maximizing social efficiency or revenue, as shown in Algorithm 1. In our framework, we can flexibly choose different optimization targets according to the practical requirements of auction problems. To ensure the worst case profit, the primary user will set a virtual reservation price $\eta^{\theta}$, which is the minimum virtual price for spectrums per unit time. Let $\{\xi\}_{\Psi}$ denote a set whose elements satisfy property $\Psi$. The details are depicted as follows.

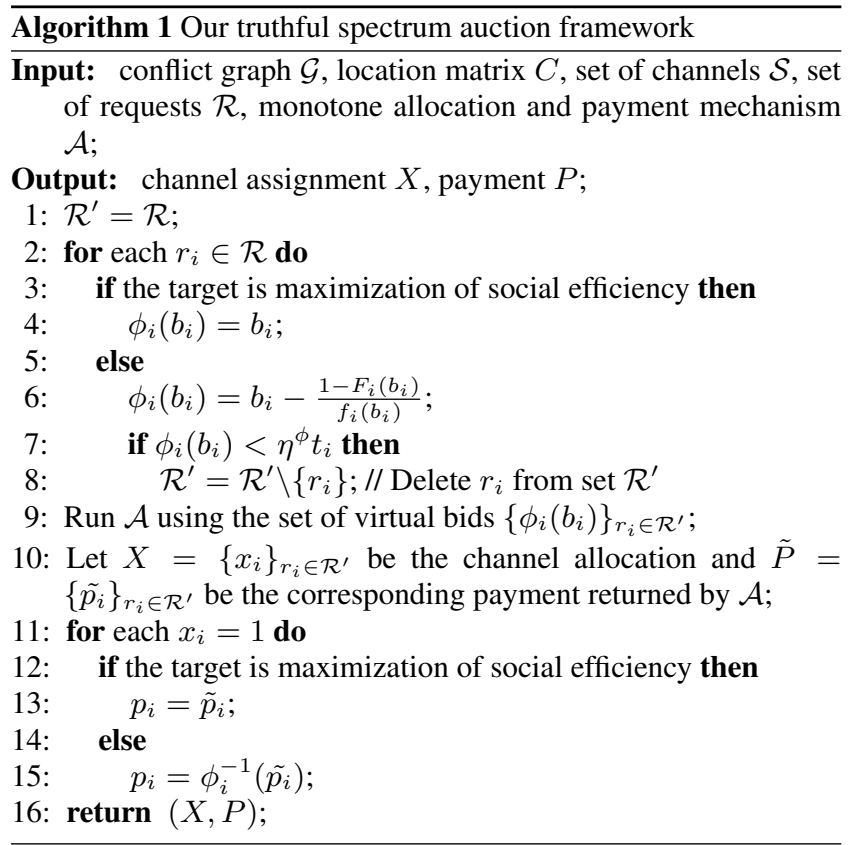

\section{ALLOCATION MECHANISM WITH AP- PROXIMATION RATIO (1-1/e)}

\subsection{The Optimal Channel Allocation}

In the channel allocation problem, we need to match the requests and channels optimally under their constraints. In order to simplify the matching model between requests and channels, we would like to segment the available time of each channel into many time slices. Recall that the available time of each channel is $[0, \mathrm{~T}]$ in each auction period. Then, we use the arrival time $a_{i}$ and deadline $d_{i}$ of each request $r_{i}$ to partition the time interval $[0, \mathrm{~T}]$. Each arrival time/deadline of requests divides the time axis of one channel into two parts. Suppose there are $n$ requests, the time interval $[0, \mathrm{~T}]$ is divided into no more than $2 n+1$ time slices.

After the introduction of segmentation process, we give the detailed description of the channel allocation problem. First, for each partitioned time slice derived from channel $s_{j}$, it can only be allocated to the requests within the license area of channel $s_{j}$. Let $x_{j, i}^{l}$ represent whether the $l$-th time slice of channel $s_{j}$ is allocated to the request $r_{i}$, then we get a constraint $x_{j, i}^{l} \leq c_{i, j}$. Second, each time slice can only be allocated to requests conflict-free with each other. Thus, we get another constraint $\sum_{k \neq i} x_{j, k}^{l} y_{i, k, j}+x_{j, i}^{l} \leq 1$. Let $t_{j}^{l}$ be the length of $l$-th time slice in channel $s_{j}$. Modify $a_{i}$ to be the first time slice $r_{i}$ wants to use, and $d_{i}$ to be the last time slice $r_{i}$ wants to use. Moreover, if we allocate request $r_{i}$ in channel $s_{j}$, 
the time assigned to request $r_{i}$ from channel $s_{j}$ should be equal to the required time of request $r_{i}$, so we get $\sum_{l=a_{i}}^{d_{i}} x_{j, i}^{l} t_{j}^{l}=t_{i} x_{i, j}$. From the analysis above, the allocation problem can be formulated as follows.

$$
\begin{aligned}
& \max \sum_{s_{j} \in \mathcal{S}} \sum_{r_{i} \in \mathcal{R}^{\prime}} \phi_{i}\left(b_{i}\right) x_{i, j}, \text { subject to } \\
& \left\{\begin{array}{l}
\sum_{s_{j} \in \mathcal{S}} x_{i, j} \leq 1, \forall r_{i} \in \mathcal{R}^{\prime} \\
x_{j, i}^{l} \leq c_{i, j}, \forall s_{j} \in \mathcal{S}, \forall r_{i} \in \mathcal{R}^{\prime}, \forall l \\
\sum_{k \neq i} x_{j, k}^{l} y_{i, k, j}+x_{j, i}^{l} \leq 1, \forall s_{j} \in \mathcal{S}, \forall r_{i} \in \mathcal{R}^{\prime}, \forall l \\
\sum_{i=a_{i}}^{d_{i}} x_{j, i}^{l} t_{j}^{l}=t_{i} x_{i, j}, \forall s_{j} \in \mathcal{S}, \forall r_{i} \in \mathcal{R}^{\prime} \\
x_{i, j} \in\{0,1\}, \forall s_{j} \in \mathcal{S}, \forall r_{i} \in \mathcal{R}^{\prime} \\
x_{j, i}^{l} \in\{0,1\}, \forall s_{j} \in \mathcal{S}, \forall r_{i} \in \mathcal{R}^{\prime}, \forall l
\end{array}\right.
\end{aligned}
$$

where $x_{i, j}$ stands for whether channel $s_{j}$ is allocated to request $r_{i}$ or not, $y_{i, k, j}$ represents whether request $r_{i}$ conflicts with request $r_{k}$ or not in channel $s_{j}$.

\section{2 (1-1/e)-Approximation methods}

$L P$ relaxation technique can be introduced to solve NP-hard problems, and it often leads to a good approximation algorithm. We release $\mathrm{IP}(1)$ to linear programming $\mathrm{LP}(2)$ by replacing $x_{i, j} \in\{0,1\}$ with $0 \leq x_{i, j} \leq 1$, and replacing $x_{j, i}^{l} \in\{0,1\}$ with $0 \leq x_{j, i}^{l} \leq 1$.

Recall that the number of time slices is no more than $2 n+1$ for each channel, so LP(2) has a polynomial number of variables and constraints, and can be solved optimally in polynomial time.

\subsubsection{Randomized Rounding}

Suppose $O_{L P 2}$ is the optimal solution of LP(2), we apply a standard randomized rounding on it to obtain an integral feasible solution $f_{I P 1}$ to IP (1). The rounding procedure is presented as follows.

1. Randomly choose a channel $s_{j}$, randomly choose a request $r_{i}$ with $x_{i, j}>0$, and set $x_{i, j}=1$;

2. If $x_{i, j}=1$, set $x_{k, j}=0$ for all requests $r_{k}$ with $y_{i, k, j}=1$;

3. If $x_{i, j}=1$, set $x_{i, k}=0$ for all channels with $k \neq j$.

4. Repeat steps 1 to 3 until all requests have been processed.

Through the randomized rounding procedure above, the optimal solution of $\mathrm{LP}(2)$ is converted into a feasible solution of IP(1). Let $w_{O_{L P 2}}$ be the weight of $O_{L P 2}$, and let $E\left(w_{f_{I P 1}}\right)$ be the expected weight of $f_{I P 1}$. We show in Theorem 1 that $E\left(w_{f_{I P 1}}\right) \geq(1-$ $1 / e) w_{O_{L P 2}}$.

THEOREM 1. The expected weight of the rounded solution is at least $1-1$ /e times of the weight of the optimal solution to LP (2).

Proof. Due to the page limit, all the detailed proofs can be referred to [6].

We have shown that the expected weight of feasible solution $f_{I P 1}$ of IP (1) obtained by our randomized rounding is larger than $1-1 / e$ times of the weight of the optimal solution of LP (2). Obviously, the weight of the optimal solution of LP (2), which is denoted by $w_{O_{L P 2}}$, is larger than the optimal solution of IP (1), which is denoted by $w_{O_{I P 1}}$. Therefore, we can get that

THEOREM 2. The expected weight of the rounded solution is at least $1-1 /$ e times of the weight of the optimal solution to IP (1).

\subsubsection{Deterministic Methods}

The rounding procedure only makes sure that the expected weight of $f_{I P 1}$ is larger than $1-1 / e$ times of the weight of $O_{L P 2}$. What we need is to find a feasible solution of $\operatorname{IP}(1)$ whose weight is exactly larger than $1-1 / e$ times of the $w_{O_{L P 2}}$. In the following, we show that the rounding procedure can be derandomized and how the method of conditional probabilities can be used in our setting.

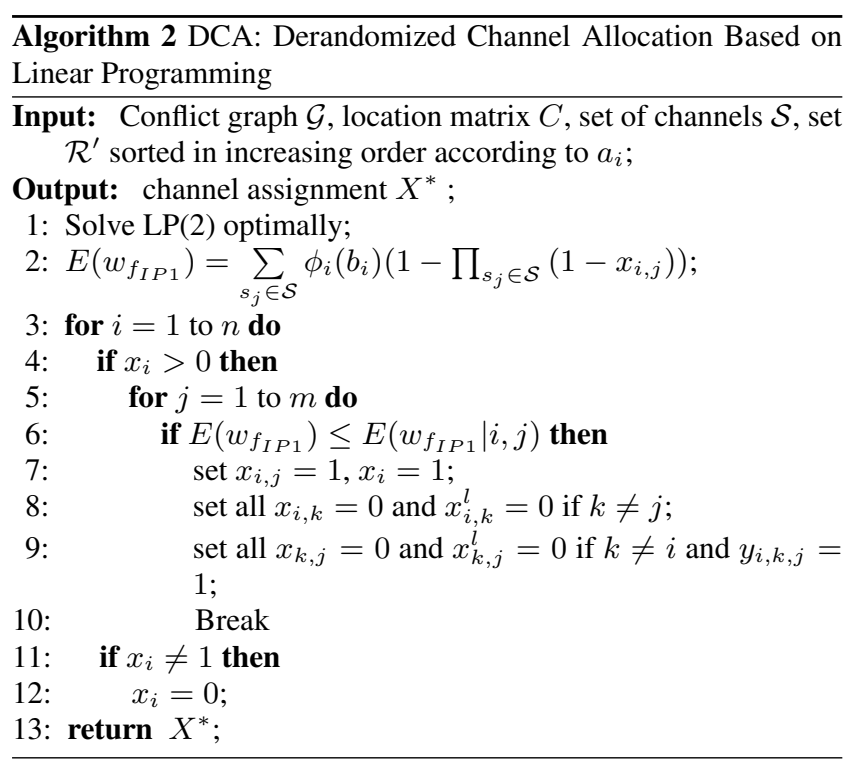

Let $E\left(w_{f_{I P 1}} \mid r_{i} \rightarrow s_{j}\right)$ be the expected weight when request $r_{i}$ is allocated in channel $s_{j}$, and let $E\left(w_{f_{I P 1}} \mid \tilde{i}\right)$ be the expected weight when request $r_{i}$ will not be allocated in any channel. Next, we will show how our derandomization algorithm works. We first sort all the requests by their arrival time $a_{i}$ in the ascending order. Let $x_{i}=\sum_{j \in \mathcal{S}} x_{i, j}$, and then scan all the requests one by one to decide which request can be allocated in channels. When request $r_{i}$ is considered, we scan all of the channels that are available for $r_{i}$ to check if $r_{i}$ can be allocated in one of them. If $E\left(w_{f_{I P 1}} \mid r_{i} \rightarrow\right.$ $\left.s_{j}\right)<E\left(w_{f_{I P 1}}\right)$, set $x_{i, j}=0$; otherwise, allocate $r_{i}$ in channel $s_{j}$, and set $x_{i, j}=1, x_{i}=1, x_{i, k}=0$ if $k \neq j$. Meanwhile, if $r_{i}$ is allocated in channel $s_{j}$, we set $x_{k, j}^{l}=0$ if $y_{i, k, j}=1$.

Suppose $r_{i}$ is the first request that satisfies $x_{i}>0$ in the ordered requests. Let $q_{i, j}$ denote the probability that request $r_{i}$ is allocated in channel $s_{j}$ and let $q_{\tilde{i}}$ denote the probability that $r_{i}$ is not allocated in any channel. By the formula for conditional probabilities, we have

$$
E\left(w_{f_{I P 1}}\right)=\sum_{r_{j} \in S} E\left(w_{f_{I P 1}} \mid r_{i} \rightarrow s_{j}\right) q_{i, j}+E\left(w_{f_{I P 1}} \mid \tilde{i}\right) q_{\tilde{i}}
$$

In particular, there exists at least one conditional expectation in $E\left(w_{f_{I P 1}} \mid r_{i} \rightarrow s_{1}\right), \cdots, E\left(w_{f_{I P 1}} \mid r_{i} \rightarrow s_{m}\right), E\left(w_{f_{I P 1}} \mid \tilde{i}\right)$, which is larger than $E\left(w_{f_{I P 1}}\right)$. If it is $E\left(w_{f_{I P 1}} \mid r_{i} \rightarrow s_{j}\right) \geq$ $E\left(w_{f_{I P 1}}\right)$, we allocate request $r_{i}$ in channel $s_{j}$; otherwise, $E\left(w_{f_{I P 1}}\right.$ $\mid \tilde{i}) \geq E\left(w_{f_{I P 1}}\right)$ holds, reject request $r_{i}$, and set $x_{i, j}=0$ for each $s_{j} \in \mathcal{S}$. This can be done since $E\left(w_{f_{I P 1}}\right)=\sum_{r_{i} \in \mathcal{R}^{\prime}} \phi_{i}\left(b_{i}\right) q_{i}$, and $q_{i}$ can be computed precisely by

$$
q_{i}=1-\prod_{s_{j} \in \mathcal{S}}\left(1-x_{i, j}\right)
$$

Let $q_{r_{i} \rightarrow s_{j}, k}$ stand for the probability that request $r_{k}$ is allocated in a channel when request $r_{i}$ is allocated in $s_{j}$. Then $q_{r_{i} \rightarrow s_{j}, k}$ can be calculated by

$$
q_{r_{i} \rightarrow s_{j}, k}=\left\{\begin{array}{l}
1-\prod_{o \neq j}\left(1-x_{k, o}\right), y_{i, k, j}=1 \\
q_{k}, \text { otherwise }
\end{array}\right.
$$


For each request $r_{i}$, we can compute $E\left(w_{f_{I P 1}} \mid r_{i} \rightarrow s_{j}\right)$ precisely as the follows

$$
E\left(w_{f_{I P 1}} \mid r_{i} \rightarrow s_{j}\right)=\phi_{i}\left(b_{i}\right)+\sum_{k \neq i} \phi_{k}\left(b_{k}\right) q_{r_{i} \rightarrow s_{j}, k}
$$

Given the selections in the prior requests, we can continue deterministically to allocate other requests and do the same thing while maintaining the invariant that the conditional expectation $E\left(w_{f_{I P 1}}\right)$, never deceases. After allocating all of the requests, we can get a feasible solution of $\operatorname{IP}(1)$ whose weight is as good as $E\left(w_{f_{I P 1}}\right)$, i.e. at least $(1-1 / e) w_{O_{L P 2}}$.

Recall that to ensure the truthfulness of our auction mechanis$\mathrm{m}$, the allocation algorithm must be bid-monotone. However, we cannot prove or disprove the bid-monotone property of the allocation method DCA (presented in Algorithm 2). Thus, it is unknown whether we can design a truthful mechanism based on this method. In the rest of the section, we revise this method and show that the revised method does satisfy the bid-monotone property.

Since that there exists at least one of the conditional expectations between $\max _{s_{j} \in \mathcal{S}} E\left(w_{f_{I P 1}} \mid r_{i} \rightarrow s_{j}\right)$ and $E\left(w_{f_{I P 1}} \mid \tilde{i}\right)$, which is larger than $E\left(w_{f_{I P 1}}\right)$. Thus, if we allocate $r_{i}$ in the channel with the maximal conditional expectation as long as $\max _{s_{j} \in \mathcal{S}} E\left(w_{f_{I P 1}} \mid\right.$ $\left.r_{i} \rightarrow s_{j}\right) \geq E\left(w_{f_{I P 1}} \mid \tilde{i}\right)$, and do not allocate $r_{i}$ in any channel otherwise, we can also get a feasible solution of IP(1), whose weight is as good as $E\left(w_{f_{I P 1}}\right)$.

This can be done since we can compute $E\left(w_{f_{I P 1}} \mid i, j\right)$ and $E\left(w_{f_{I P 1}} \mid \tilde{i}\right)$ precisely as follows:

$$
E\left(w_{f_{I P 1}} \mid r_{i} \rightarrow s_{j}\right)=\phi_{i}\left(b_{i}\right)+E_{k \neq i}\left(w_{f_{I P 1}^{\prime}} \mid r_{i} \rightarrow s_{j}\right)
$$

where $E_{k \neq i}\left(w_{f_{I P 1}^{\prime}} \mid r_{i} \rightarrow s_{j}\right)$ is the expected weight of all other requests when request $r_{i}$ has been allocated in channel $s_{j}$. We can get it by allocating $r_{i}$ in channel $s_{j}$ first, and then solve LP(2) optimally with other requests.

$$
E\left(w_{f_{I P 1}} \mid \tilde{i}\right)=E_{\mathcal{R}^{\prime} / r_{i}}\left(w_{f_{I P 1}^{\prime}}\right)
$$

where $E_{\mathcal{R} / r_{i}}\left(w_{f_{I P 1}^{\prime}}\right)$ is the expected weight of all other requests when request $r_{i}$ is not allocated in any channel. We can get it by solving LP(2) optimally with requests except $r_{i}$.

Based on the observation above, we give a revised version (called MDCA) of Algorithm DCA as follows.

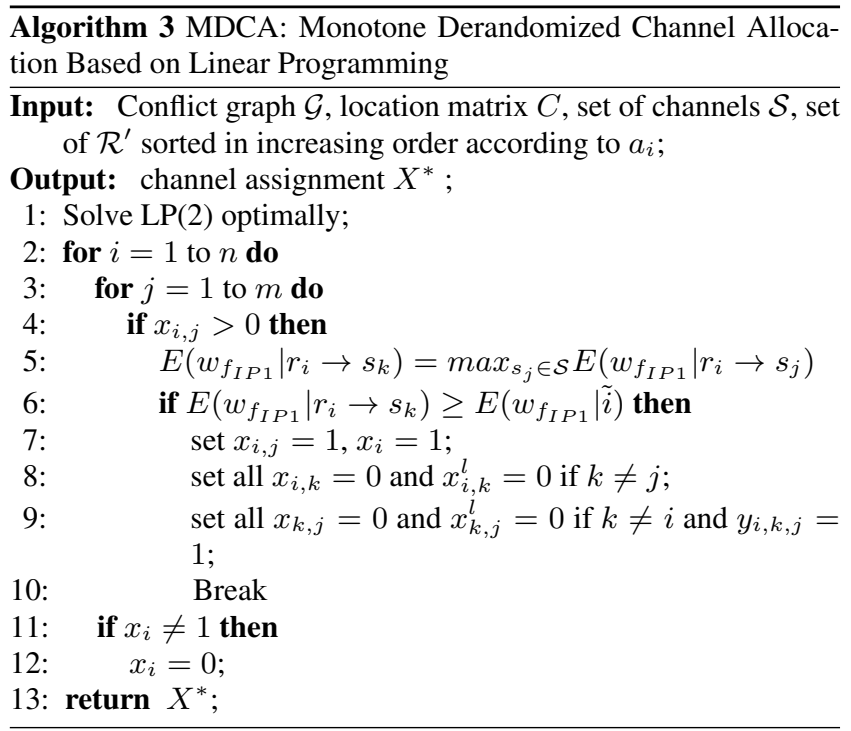

In MDCA, we first sort all of the requests by their arrival times in the ascending order, and then we scan all requests one by one to decide which request can be allocated in channels. When request $r_{i}$ is considered, we compute $E\left(w_{f_{I P 1}} \mid r_{i} \rightarrow s_{j}\right)$ for all channels $s_{j} \in \mathcal{S}$ that no request conflicting with it has been allocated in. We allocate $r_{i}$ in channel $s_{k}$ when $E\left(w_{f_{I P 1}} \mid r_{i} \rightarrow s_{k}\right)=$ $\max _{s_{j} \in \mathcal{S}} E\left(w_{f_{I P 1}} \mid r_{i} \rightarrow s_{j}\right) \geq E\left(w_{f_{I P 1}} \mid \tilde{i}\right)$, and reject it otherwise. After the last request was considered in MDCA, we get a feasible solution of IP (1), whose weight is as good as $E\left(w_{f_{I P 1}}\right)$.

\section{THEOREM 3. MDCA (see Algorithm 3) is bid monotone.}

Since the revised Algorithm MDCA is bid-monotone, there exists a critical value for each winner. Thus, we can design a truthful auction mechanism through charging each winner its critical value.

\section{CONCLUSION}

In this paper, we have studied the case that spectrum can be reused spatial domain, temporal domain. We have designed a general truthful spectrum auction framework which can maximize the social efficiency or revenue. As allocating channels optimally is NP-hard in our model, we have also proposed a set of near-optimal channel allocation mechanisms with an approximation factor of $(1-1 / e)$.

\section{Acknowledgement}

The research of authors is partially supported by the National Grand Fundamental Research 973 Program of China (No.2011CB302905, No.2011CB302705), National Natural Science Foundation of China (NSFC) under Grant No. 61202028, No. 61170216, No. 612282 02, and NSF CNS-0832120, NSF CNS-1035894, NSF ECCS-1247 944. Specialized Research Fund for the Doctoral Program of Higher Education (SRFDP) under Grant No. 20123201120010.

\section{REFERENCES}

[1] L. Deek, X. Zhou, K. Almeroth, and H. Zheng. To preempt or not: Tackling bid and time-based cheating in online spectrum auctions. In IEEE INFOCOM 2011, pages 2219-2227, 2011.

[2] M. Dong, G. Sun, X. Wang, and Q. Zhang. Combinatorial auction with time-frequency flexibility in cognitive radio networks. In IEEE INFOCOM 2012, pages 2282-2290, 2012.

[3] A. Gopinathan, Z. Li, and C. Wu. Strategyproof auctions for balancing social welfare and fairness in secondary spectrum markets. In IEEE INFOCOM 2012, pages 2813-2821, 2011.

[4] R.B. Myerson. Optimal auction design. Mathematics of operations research, 6(1):58-73, 1981.

[5] N. Nisan. Algorithmic game theory. Cambridge Univ Pr, 2007.

[6] Yu-e Sun, He Huang, Xiang-Yang Li, Zhili Chen, Wei Yang, Hongli $\mathrm{Xu}$, and Liusheng Huang. Near-optimal truthful auction mechanisms in secondary spectrum markets. arXiv preprint arXiv:1305.6390, 2013.

[7] H. Xu, J. Jin, and B. Li. A secondary market for spectrum. In IEEE INFOCOM 2010, pages 1-5, 2010.

[8] P. Xu and X.Y. Li. TOFU: semi-truthful online frequency allocation mechanism for wireless network. IEEE/ACM Transactions on Networking (TON), 19(2):433-446, 2011.

[9] P. Xu, X.Y. Li, and S. Tang. Efficient and strategyproof spectrum allocations in multichannel wireless networks. IEEE Transactions on Computers, 60(4):580-593, 2011.

[10] P. Xu, S.G. Wang, and X.Y. Li. SALSA: Strategyproof online spectrum admissions for wireless networks. IEEE Transactions on Computers, 59(12):1691-1702, 2010.

[11] X. Zhou, S. Gandhi, S. Suri, and H. Zheng. ebay in the sky: strategy-proof wireless spectrum auctions. In ACM Mobicom 2008, pages 2-13, 2008. 